\title{
Transfer across modality in perceptual implicit memory
}

\author{
DIANA BLUM and ANDREW P. YONELINAS \\ University of California, Davis, California
}

\begin{abstract}
We examined whether words studied in one modality (visual or auditory) would prime performance in the opposite modality in five different perceptual implicit memory tests: auditory perceptual identification, auditory stem completion, visual perceptual identification, visual stem completion, and visual fragment completion. Significant transfer across modality was observed in all five tasks. However, a large proportion of the subjects reported using explicit retrieval strategies during the implicit tests. Those subjects who claimed not to have used explicit retrieval processes during the test phase demonstrated transfer across modalities in the stem completion tests and the perceptual identification tests, but not in the fragment completion test. The results indicate that implicit visual word-fragment completion is unique, in the sense that it relies exclusively on perceptual memory processes, whereas the other tasks rely, in part, on nonperceptual memory processes.
\end{abstract}

Performance on implicit memory tests is functionally distinct from performance on explicit tests. For example, changes in modality between study and test, such as those from auditory to visual, greatly reduce priming in implicit tests, such as stem completion, fragment completion, and perceptual identification (for reviews, see Kirsner, Dunn, \& Standen, 1989; Roediger \& McDermott, 1993; Schacter, Chiu, \& Ochsner, 1993). In contrast, modality often does not affect explicit tests, such as recall and recognition. The sensitivity of the implicit memory tests to changes in perceptual format has been taken as showing that these tests rely on data-driven processes or perceptual memory systems (Jacoby, 1983; Roediger \& Blaxton, 1987; Schacter, 1992; Tulving, 1985). However, significant priming effects are observed even when the study and the test stimuli are presented in different modalities, and these results suggest that implicit memory tasks rely, in part, on modality-independent processes. For example, transfer across modality has been taken as evidence that perceptual implicit memory relies on phonological/exical processes (e.g., Curran, Schacter, \& Galluccio, 1999; Kirsner et al., 1989; Rueckl \& Mathew, 1999 ) or semantic processes (e.g., Bassili, Smith, \& MacLeod, 1989).

However, Jacoby, Yonelinas, and Jennings (1997) argued that transfer across modality in tasks like wordfragment completion was due to the fact that performance on the implicit memory tasks was contaminated by the use of explicit memory retrieval strategies. They found that in standard word-fragment completion condi-

The research was supported by Grant MH59352-01 from the National Institute of Mental Health. Correspondence concerning this article should be addressed to A. P. Yonelinas, Department of Psychology, University of California, Davis, CA 95616 (e-mail: apyonelinas@ucdavis.edu). tions, there was significant transfer across modality, but when the use of explicit memory was decreased by dividing attention at encoding, the effect was greatly reduced. Moreover, subjects were questioned after the test was completed, and it was found that subjects who were aware that they could use items from the study list to complete the test fragments exhibited transfer across modality but subjects who were not aware of this relationship did not. Those authors also used the process dissociation procedure to estimate the conscious and automatic influences of memory and found that transfer across modality in the fragment completion task could be attributed completely to the conscious use of memory.

The aim of the present study was to determine whether priming was observed across modality in a variety of perceptual implicit memory tests, when the effects of explicit memory contamination were minimized. Although transfer across modality in the fragment completion test appears to be due to explicit contamination, the transfer seen in other implicit tasks may not be due entirely to contamination. For example, in a visual word-stem completion test, subjects may pronounce the word stem when they are attempting to solve it, and this phonological information may match that encoded earlier when the word was heard in the study list (see Kirsner et al., 1989). This phonological match may support priming across modality even when subjects are not using explicit memory (see Hirshman, Passanante, \& Arndt, 1999). Similarly, in an auditory word-stem completion task, subjects may exhibit transfer from visually studied words if they engaged in phonological processing of the word when it was studied.

Determining whether priming across modality is due to explicit contamination is essential in order to fully characterize the processes underlying implicit memory and, ultimately, in order to identify the cortical regions that underlie these processes (Moscovitch, 1994; Schacter et al., 
1993; Tulving \& Schacter, 1990). If transfer across modality is due to explicit contamination, this would suggest that this form of implicit memory is based on purely perceptual processes. In contrast, if these effects are not due to contamination, this would suggest that more abstract processes, such as phonological or semantic processes, must be involved.

We examined transfer across modality in five experiments. In each experiment, the subjects studied one list of words visually and another list auditorially. During the study phase, the subjects were engaged in a continuous distractor task (counting backward out loud). The distractor task served to reduce the subjects' ability to later explicitly recollect the study words. Moreover, it served to prevent the subjects from overtly articulating each word during encoding. If they overtly pronounced a visually presented word during study, the item would be studied both visually and auditorially, and thus it could not be used to measure transfer across modality.

After the study phase, the subjects were given a distractor task, followed by one of five different implicit memory tests. In Experiment 1, the subjects were tested with an auditory perceptual identification task in which words were presented in a background of noise and the subject was required to identify each word. In Experiment 2 , the subjects received an auditory stem completion test in which the initial few phonemes of each word were presented and they were required to complete the stems with the first word that came to mind. In Experiment 3 , a visual perceptual identification task was used, in which words were presented briefly on a computer screen and the subjects were required to identify each word. In Experiment 4, the subjects were tested with a visual stem completion task in which the initial few letters of each word were presented and the subjects were required to complete the stems with the first word that came to mind. Because the results of Experiments 1-4 were inconsistent with the visual fragment completion results reported by Jacoby et al. (1997), a final experiment was conducted using a fragment completion test to ensure that the effects we observed were not related to the specific words that we had selected.

In addition to using a divided attention manipulation at the time of encoding to reduce the influence on explicit memory, the subjects in all the experiments were asked whether they had become aware that the test items could be completed with studied words and whether they had used explicit retrieval strategies during the test. In this way, we could remove those subjects who reported using explicit retrieval strategies. Such subjective reports should be interpreted cautiously, because it is possible that some subjects may fail to report when they are using such retrieval strategies. However, numerous studies of implicit memory have shown that separating the subjects on the basis of these reports is important, because performance can differ dramatically between the subjects who do and those who do not report using explicit retrieval strategies (see, e.g., Bowers \& Schacter, 1990; McKone \& Slee, 1997; Richardson-Klavehn, Lee, Joubran, \& Bjork, 1994).

Because the methods and analyses were similar for all five experiments, all of the experiments will be described together.

\section{EXPERIMENTS 1-5}

\section{Method}

Subjects. One hundred and twenty-two students (24 in each of Experiments 1-4, and 26 in Experiment 5) from the University of California at Davis participated for extra credit in an introductory psychology course.

Materials. Sixty-nine words, six to eight letters in length, were divided into three lists. Each item formed a unique word stem and word fragment (see the Appendix). Each list was either presented visually or auditorially or served as a new item list. Word lists were counterbalanced so that each list served equally often in each condition. The test lists contained a random mixture of all the studied items and the new items. Eighty-four additional words that were similar in length were used as practice items in the titration phase of the perceptual identification tasks.

Design and Procedure. During the study phase, the subjects were presented with a visual list of 23 words on a computer monitor and an auditory list of another 23 words on a tape recorder. Half the subjects received the visual and auditory lists in the reverse order. The words were presented one at a time, and the subjects were required to make a computer keyboard response indicating whether or not each presented word represented an object that could be held in one hand. This encoding task was selected because it did not lead the subjects to overtly articulate the study words, as appeared to be the case with several other encoding tasks that we piloted. The subjects were told that their responses were being recorded, but in fact they were not. During the presentation of the study lists, the subjects were required to count backward aloud, from 10 to 1 repeatedly, while making responses to the presented words. The subjects were asked to pay equal attention to both tasks and were told that each time they made a mistake or paused, it would be recorded as an error.

After the presentation of the study lists, the subjects were asked whether they had heard themselves pronounce the words while counting backward. The subjects' performance on the later memory tests was not related to their responses to this question and thus will not be discussed further. The subjects then worked on a logic puzzle as a distractor task for 5 min before beginning the test phase.

In Experiment 1, just prior to the test, the subjects were presented with the titration phase of the auditory perceptual identification task, to determine the noise level at which each subject could identify approximately $50 \%$ of the presented words. The subjects were presented with sets of eight recorded words, one word at a time, and were instructed to try to identify each word, guessing if they were not sure. A constant level of static noise from an AM radio was presented in order to make the word identification task difficult. The radio and the speaker used for the presentation of the auditory word lists were in front of the subject. After each block of eight words, the noise level was increased slightly, and the procedure was repeated with a new set of eight words. The process was repeated until each subject could correctly identify no more than four out of the eight words in a block. The subjects were then presented with the auditory perceptual identification task, using the noise level determined in the titration phase. The subjects were presented with a 
mixture of studied and nonstudied items in a background of static noise and were instructed to identify each word. As in the titration phase, they were instructed to guess if they were unsure about an item. The subjects were given $5 \mathrm{sec}$ to respond verbally to each item.

During the test phase in Experiment 2, the subjects were presented with a set of multiple completion auditory word stems and were told to complete the stems with the first word that came to mind. The word stems were recordings of the same person who had spoken the words in the study list pronouncing the first few phonemes of each word (see the Appendix). Note that the stems were pronounced in a manner that was similar to that in which the entire word had been spoken. However, the exact auditory stimuli may have differed slightly between study and test. In the test phase, one stem was presented every $5 \mathrm{sec}$. The subjects responded verbally, and the experimenter recorded each response.

In Experiment 3, just prior to the test phase, the subjects were presented with the titration phase for the visual perceptual identification task. For each trial, a string of eight ampersands (\&\&\&\&\&\&\&\&) were presented on the screen for $500 \mathrm{msec}$, followed by a word for $112 \mathrm{msec}$, followed by a mask of eight ampersands for 2,000 msec. The subjects were presented with sets of 10 words, 1 word at a time, and were instructed to say each identified word aloud, guessing if they were not sure. After each block of 10 words, the presentation duration of the target words was decreased by $17 \mathrm{msec}$, and the procedure was repeated with a new set of 10 words. The process was repeated until each subject correctly identified no more than 5 out of the 10 words in a block. The subjects were then presented with the visual perceptual identif ication task, using the presentation rate from the titration phase. The subjects were presented with a mixture of studied and nonstudied items and were instructed to identify each word, guessing if they were not sure. The subjects were given $5 \mathrm{sec}$ to respond to each item.

During the test phase of Experiment 4, multiple completion word stems (i.e., the first few letters of a word) were presented visually on a computer monitor (see the Appendix), and the subjects were instructed to complete each stem with the first word that came to mind. The subjects were given $5 \mathrm{sec}$ to respond verbally to each item.

During the test phase of Experiment 5, single completion word fragments were presented visually on a computer monitor, and the subjects were instructed to complete each fragment with the first word that came to mind. As in Jacoby et al. (1997), the word fragments did not include complete syllables; thus, they were not easily pronounceable (see the Appendix). The subjects were given $5 \mathrm{sec}$ to respond verbally to each item. Note that 26 subjects completed the fragment completion test, rather than the 24 that were required to fill the counterbalanced order. Rather than discard the data from the last 2 subjects, their data were included. Subsequent analysis showed that the counterbalancing factors did not significantly influence performance.

At the completion of the test phase in each experiment, the subjects were asked whether they were aware that some of the items in the test phase could be completed by items in the study phase (e.g., While doing the stem completion test, did you notice whether you completed some of the stems with words studied earlier in the experiment?) and whether they had explicitly attempted to use memory for those items during the test phase (e.g., Did you try to use words from earlier in the experiment to complete the stems?). Approximately half of the subjects reported that they did not intentionally use explicit memory, and a subsequent analysis examined priming across modality in this noncontaminated group. Note that because there were only a small number of subjects (as few as 2 in an experiment) who were aware and who did not use explicit memory, a separate analysis based on test awareness was not conducted.

\section{Results}

Table 1 presents the average proportion of items correctly completed in the stem and fragment completion tasks and the proportion of correctly identified items in the perceptual identification tasks for Experiments 1-5. Priming was measured by comparing the probability of completing or identifying a studied item with the probability of completing or identifying a new item.

Experiment 1: Auditory perceptual identification. For the auditory perceptual identification test, there was a significant priming effect for the words that had been seen earlier $[t(23)=6.499, p<.001]$, showing that there was transfer across modalities. There was also a significant priming effect observed for the words that had been heard earlier $[t(23)=9.505, p<.001]$, and this effect was greater than that for the seen words $[t(23)=5.061, p<$ $.001]$. This latter effect indicates that there was a priming advantage when the study and the test modalities matched.

However, 15 of the 24 subjects reported that they had intentionally used explicit memory during the test phase. In order to assess priming effects that were not contaminated by the use of explicit memory strategies, priming was examined in the nonintentional subjects (see Figure 1). The nonintentional subjects exhibited a significant priming effect for the seen words $[t(8)=3.464, p<.001]$, indicating that there was significant transfer across modality in the auditory perceptual identification task even for those subjects who indicated that they did not use explicit memory retrieval strategies. There was also a significant priming effect of the words that had been heard at study $[t(8)=4.007, p<.001]$. This effect was marginally greater than the priming effect of words that had been seen earlier $[t(8)=1.721, p<.062]$.

Experiment 2: Auditory stem completion. For the auditory stem completion task, there was a significant priming effect for the seen words $[t(23)=5.953, p<$ $.001]$, indicating transfer across modality. There was

Table 1

Overall Perceptual Implicit Memory Performance

\begin{tabular}{clccc}
\hline & \multicolumn{1}{c}{ Test } & \multicolumn{3}{c}{ Encoding Condition } \\
\cline { 3 - 5 } Experiment & \multicolumn{1}{c}{ Auditory } & Visual & New \\
\hline 1 & Auditory perceptual identification & .63 & .48 & .32 \\
2 & Auditory stem completion & .26 & .21 & .11 \\
3 & Visual perceptual identification & .52 & .67 & .39 \\
4 & Visual stem completion & .34 & .44 & .21 \\
5 & Visual fragment completion & .25 & .39 & .18 \\
\hline
\end{tabular}



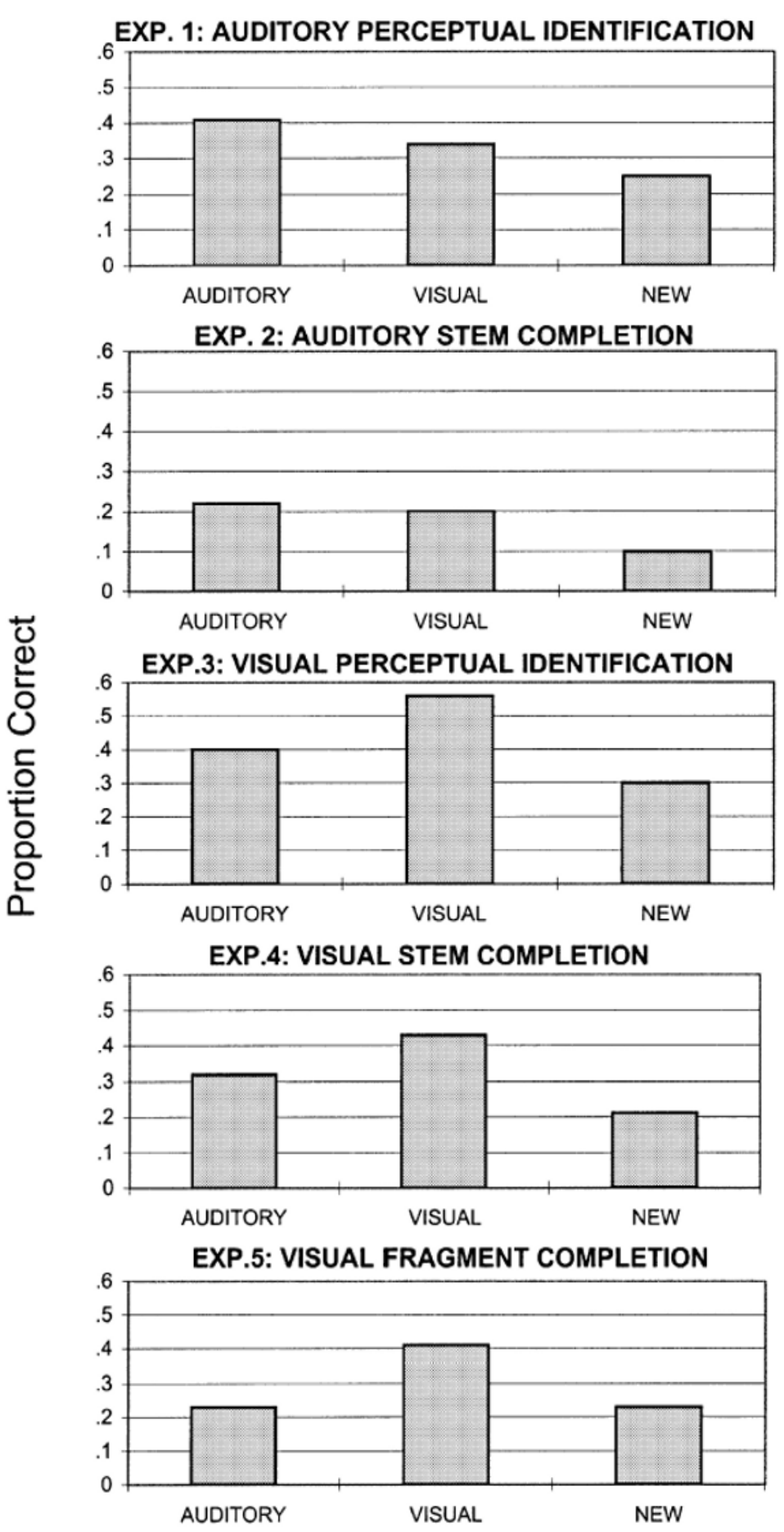

Figure 1. Implicit memory performance as a function of encoding condition for subjects who did not report using intentional memory retrieval strategies.

also a significant priming effect for the heard words $[t(23)=6.711, p<.001]$, and this priming effect was larger than that for the seen words $[t(23)=1.913, p<$ $.05]$. However, 11 of the 24 subjects reported that they had intentionally used explicit memory during the test phase. The nonintentional subjects (see Figure 1) exhibited significant priming effects for the seen words [ $t$ (12) $=3.838, p<.001]$, indicating that transfer across modality in the auditory stem completion task was observed even in the subjects who did not report intentionally 
using explicit memory. There was also a significant priming effect for the words that had been heard at study $[t(12)=4.635, p<.001]$. This effect was not significantly greater then that observed for the seen words $[t(12)=$ $0.987, p<.172$ ], suggesting that the modality match between study and test did not influence priming in the auditory stem completion test. The latter finding will be discussed after the presentation of the results from the following experiments.

Experiment 3: Visual perceptual identification. In the visual perceptual identification task, there was significant priming for the heard words $[t(23)=3.832, p<$ $.001]$, suggesting that there was transfer across modality. There was also a significant priming effect for the seen words $[t(23)=7.598, p<.001]$, and this effect was greater than that observed for the heard words $[t(23)=3.835$, $p<.001]$. However, 17 of the subjects reported that they used explicit memory retrieval strategies during the test phase. The nonintentional subjects (see Figure 1) exhibited a significant priming effect for the heard words $[t(6)=2.931, p<.05]$, showing that there was significant transfer across modality for subjects who did not report using explicit memory retrieval strategies. There was also significant priming for the seen words $[t(6)=$ $3.576, p<.05]$, and the priming effect was marginally greater for the seen than for the heard words $[t(5)=$ $1.815, p<.065]$.

Experiment 4: Visual stem completion. In the visual stem completion task, there was significant priming for the heard words $[t(23)=5.297, p<.001]$ and for the seen words $[t(23)=9.559, p<.001]$. Furthermore, the priming effects were greater for the seen than for the heard words $[t(23)=3.318, p<.01]$. However, 6 of the subjects reported that they used explicit memory retrieval strategies during the test phase. The nonintentional subjects (see Figure 1) exhibited a significant priming effect for the heard words $[t(17)=4.069, p<.001]$, indicating that there was significant transfer across modality in the visual stem completion task for the subjects who did not report using explicit memory retrieval strategies. There was also significant priming for seen words $[t(17)=$ $7.558, p<.001]$, and the priming effects were greater for the seen than for the heard words $[t(17)=2.602, p<.01]$.

The results of Experiments 1-4 showed that there was significant transfer across modality for the auditory and visual stem completion tests, as well as for the auditory and visual perceptual identification tests, even when only the performance of the nonintentionalsubjects was examined. These results are in disagreement with those reported by Jacoby et al. (1997), who found that there was no transfer across modality in word-fragment completion tests when the use of explicit retrieval was eliminated. In order to determine whether this discrepancy was due to differences in the materials used in the two studies, in Experiment 5, we examined visual fragment completion, using the same word lists as those used in Experiments 1-4.
Experiment 5: Visual fragment completion. In the visual fragment completion task, there was significant priming for the heard words $[t(25)=3.413, p<.002]$, indicating transfer across modality. There was also significant priming for the seen words $[t(25)=10.143, p<$ $.0001]$, and this priming effect was greater than that observed for the heard words $[t(25)=5.14, p<.001]$. However, 16 of the 26 subjects reported that they used explicit memory retrieval strategies during the test phase. Unlike the previous four experiments, the nonintentional subjects (see Figure 1) did not show a significant priming effect for the heard words $[t(9)=0.190, p>.1]$. In fact, an examination of Figure 1 shows that the subjects were as likely to produce a new word as they were to produce a heard word. Thus, the present results replicate those of Jacoby et al. (1997) in showing that transfer across modality in the fragment completion test was due to the subjects who reported using intentional retrieval strategies. For the nonintentional subjects, there was a significant priming effect for the seen words $[t(9)=$ $5.658, p<.0001]$, and this priming effect was greater than that for the heard words $[t(9)=2.82, p<.01]$.

\section{Discussion}

Is transfer across modality in perceptual implicit memory tests due to the influence of intentional retrieval strategies? The present results indicate that the answer to this question depends on the specific memory task. Transfer across modality was consistently observed in stem completion and perceptual identification tasks, but it was only observed in the fragment completion task for those subjects who reported using intentional retrieval strategies. When overall priming scores were examined, significant transfer across modality was observed in the auditory and visual stem completion tasks and in the auditory and visual perceptual identification tasks. Importantly, the conclusions did not change when the subjects who used intentional retrieval strategies were removed from the analyses. Transfer across modality has been observed in numerous previous studies (see Roediger \& McDermott, 1993). However, these earlier studies often did not use such manipulations as dividing attention during study as a way of reducing explicit memory, and they generally did not assess whether subjects used intentional memory retrieval strategies, so it was difficult to determine whether the observed transfer across modality was due to the use of explicit memory. The present results show that transfer across modality persisted in these tests even when the contribution of explicit retrieval was minimized.

In the fragment completion test, on the other hand, there was transfer across modality when overall priming scores were examined, but the effect was due to the subjects who reported using explicit memory retrieval strategies; there was no evidence of transfer across modalities for the nonintentionalimplicit subjects. These results are consistent with those of Jacoby et al. (1997), who found that 
transfer across modality in the fragment completion test was due to subjects who reported using explicit memory.

There are several procedural differences between the five implicit memory tests examined in the present study that may have influenced performance, but they do not account for the fact that transfer across modality was only absent in the fragment completion test. First, baseline levels of performance (i.e., performance on the new items) varied across the experiments, and this may have influenced the magnitude of the observed priming effects. However, the baseline in the fragment completion test was not the highest or the lowest of the five tests, and it was close to that obtained in the visual stem completion test (see Table 1). Second, if the overall priming effects in the fragment completion test were lower than those in all of the other tests, floor effects might account for the lack of transfer effects in the fragment test. However, the overall priming effects were actually lower in the auditory stem completion test (.12) than in the fragment completion test (.14), and significant transfer effects were observed in that stem completion test. Third, unlike the fragment completion experiment, the subjects received practice in the titration phases of the visual and auditory perceptual identification tasks, and this may have influenced performance. However, the subjects did not receive practice in the auditory or visual stem completion tests, showing that practice in itself was not a critical factor in predicting transfer across modality.

Was the failure to find significant transfer across modality in the fragment completion test related to insufficient statistical power? In that experiment, only 10 of the subjects claimed to complete the implicit test without using intentional retrieval strategies; thus, it is quite possible that with more subjects, evidence for transfer across modality might be detected. However, significant priming effects across modality were observed in Experiments 1 and 3, which had even fewer nonintentional subjects. Moreover, as will be discussed below, the present findings are supported by several other studies.

Removing the intentional subjects from the analysis, as was done in the present experiments, means that the full counterbalancing was disrupted, and this may have had an impact on the results. ${ }^{1}$ Subsequent analyses, however, suggested that the results were not critically influenced by the counterbalancing factors. In order to determine whether there were differences in the magnitude of the priming effects in the three different counterbalancing orders of words that we used, we conducted item analyses and found that the priming effects did not differ as a function of the stimulus sets in the fragment completion, visual stem completion, auditory stem completion, or visual perceptual identification experiments. Only in the auditory perceptual identification experiment was there a significant effect of item $[F(2,21)=5.392, p<.05]$, reflecting the fact that the priming effects were larger in one of the three stimulus orders. However, this difference did not influence the priming effects of the nonintentional group, because as it turned out, there were equal numbers of nonintentional subjects in each of the item counterbalances in that experiment. Additional analyses examined whether the order of the visual and auditory study lists influenced performance and showed that the priming effects were not influenced by the specific study order in any of the experiments.

It is possible that classifying the subjects on the basis of subjective reports does not completely rule out the effects of explicit retrieval. Thus, the results of studies using subjective reports, such as the present experiments, must be interpreted cautiously, and it is important to look for convergent results from other studies, as well as looking to results from studies that use alternative research methods. Support for the present results come from a number of such convergent findings. For example, in agreement with the present results, Jacoby et al. (1997) used subjective reports, as well as the process dissociation procedure, and found that transfer across modality in a wordfragment completion task was due to subjects who used explicit memory. Moreover, amnesic patients exhibit normal within-modality priming effects on fragment completion tests but do not exhibit transfer across modality in this task (Kohler et al., 1997; Vaidya, Gabrieli, Keane, \& Monti, 1995). Because these patients have severe impairments in explicit memory, their performance should provide a relatively pure measure of implicit memory, and their results suggest that transfer across modality in the fragment completion test is only observed when explicit memory contributes to performance.

The finding of significant transfer across modality observed in the visual stem completion test also converges with results from other experimental methods. For example, Jacoby, Toth, and Yonelinas (1993) examined visual stem completion, using the process dissociation procedure, and found that words that were heard during study led to a priming effect in the unconscious memory component even when the effects of conscious recollection were removed. ${ }^{2}$ Moreover, Graf, Shimamura, and Squire (1985) found that in a visual stem completion test, amnesic patients exhibited normal priming effects for words that were heard and seen during study, suggesting that transfer across modality in the stem completion task does not rely on explicit memory.

The difference between performances on the fragment completion and the other visual implicit tests suggests that these tests rely on different memory processes. The visual stem completion and visual perceptual identification tests appear to rely on both visual and nonvisual memory processes. Both tasks led to greater priming when the study items were visually processed, as compared with the cases in which they were heard, indicating that visual processing played an important role in the priming that was observed in these tasks. However, these tasks also exhibited significant transfer across modality, indicating the contribution of nonvisual processes. These nonvisual processes may be phonological in nature (see, e.g., Kirsner et al., 1989; Rueckl \& Mathew, 1999). That is, a visual word stem or rapidly presented word in a vi- 
sual perceptual identification test may be sufficient to support phonological processing of the item (e.g., the overt or covert pronunciation of part of the word), and that phonological cue may match the phonological processing of the word when it was heard in the study list. Note that it is also possible that the transfer across modality reflected semantic, rather than phonological, memory processes (see, e.g., Bassili et al., 1989; Masson \& Freedman, 1990). For example, processing the meaning of a heard word may have led to priming on the visual stem completion test. However, if this were true, it is not clear why transfer across modality was not observed in the fragment completion test. Moreover, the fact that these tasks are relatively insensitive to semantic manipulations (see, e.g., Roediger \& McDermott, 1993; Schacter \& Church, 1992) suggests that semantic processes do not play a major role in these tests.

In contrast, the visual fragment completion test appeared to rely exclusively on visual memory processes. Significant word-fragment priming in the nonintentional subjects was observed when the words were visually presented during study, but not when they were heard, indicating that visual processing at study was essential for priming in this task. Thus, the fragment completion test was the only perceptual implicit memory test that we examined that relied entirely on perceptual implicit memory processes.

The reason that the fragment completion test relied exclusively on visual perceptual processes may be that it provided exclusively visual retrieval cues. That is, the word fragments did not include complete syllables, and thus the test stimuli were not easily pronounceable. In this way, the test stimuli provided only visual retrieval cues. In contrast, the word stems formed the first syllable of each word, and thus they could be pronounced. In this way, the subjects could use either the visual cue or the phonological cue as a basis for memory retrieval. Similarly, in the perceptual identification task, the subjects may have perceived a portion of a test word that could be pronounced, and thus the test stimuli could have provided the subjects with visual as well as phonological retrieval cues.

The auditory implicit tests appear to rely on phonological, and possibly acoustic, processes. Significant transfer across modality was observed in the auditory stem and auditory perceptual identification tests. Thus, an auditory word stem or a spoken word masked in noise can serve as a cue for words that had been seen earlier. This could occur if visually presented study words led to phonological processing of the items at the time of study. There is, in fact, evidence that phonological processes are automatically engaged when words are identified (e.g., Perfetti \& Bell, 1991). There is also evidence in the present study that more perceptual processes, possibly acoustic in nature, may contribute to performance in at least some auditory implicit tests. That is, there was slightly greater priming for the heard than for the seen items in the auditory perceptual identification task, indicating the importance of a perceptual match in this task. In the auditory stem completion test, there was very little evidence of such a modality match effect; however, this lack of a modality effect could be due to the fact that the pronunciation of the word stems may have been slightly different from the pronunciation of the original auditory study words. Thus, any conclusions regarding the acoustic contribution to auditory implicit tests must await further investigation.

In conclusion, the results showed that some, but not all, perceptual implicit memory tests support transfer across modality. This suggests that not all perceptual implicit tests are alike in terms of the memory processes that they rely on. For the nonintentional subjects, the visual fragment completion task was unique, in the sense that it relied exclusively on perceptual implicit processes, whereas the stem completion and the perceptual identification tasks relied, in part, on more abstract processes.

\section{REFERENCES}

Bassili, J. N., Smith, M. C., \& MacLeod, C. M. (1989). Auditory and visual word-stem completion: Separate data-driven and conceptually driven processes. Quarterly Journal of Experimental Psychology, 41A, 439-453.

Bowers, J. S., \& Schacter, D. L. (1990). Implicit memory and test awareness. Journal of Experimental Psychology: Learning, Memory, \& Cognition, 16, 404-416.

Curran, T., Schacter, D. L., \& Galluccio, L. (1999). Cross-modal priming and explicit memory in patients with verbal production deficits. Brain \& Cognition, 39, 133-146.

Graf, P., Shimamura, A. P., \& Squire, L. R. (1985). Priming across modalities and priming across category levels: Extending the domain of preserved function in amnesia. Journal of Experimental Psychology: Learning, Memory, \& Cognition, 11, 386-396.

Hirshman, E., Passanante, A., \& Arndt, J. (1999). The effect of midazolam on the modality-match effect in implicit memory. Cognitive Brain Research, 7, 473-479.

JACOBY, L. L. (1983). Perceptual enhancement: Persistent effects of experience. Journal of Experimental Psychology: Learning, Memory, \& Cognition, 9, 21-38.

JACoby, L. L., Toth, J. P., \& Yonelinas, A. P. (1993). Separating conscious and unconscious influences of memory: Measuring recollection. Journal of Experimental Psychology: General, 122, 139-154.

JACOBY, L. L, Yonelinas, A. P., \& Jennings, J. M. (1997). The relationship between conscious and unconscious (automatic influences): A declaration of independence. In J. D. Cohen \& J. W. Scholar (Eds.), Scientific approach to the question of consciousness: The 25th annual Carnegie Symposium on Cognition (pp. 13-47). Hillsdale, NJ: Erlbaum.

Kirsner, K., Dunn, J., \& Standen, P. (1989). Domain specific resources in word recognition. In S. Lewandowsky, J. C. Dunn, \& K. Kirsner (Eds.), Implicit memory: Theoretical issues (pp. 99-122). Hillsdale NJ: Erlbaum.

Kohler, S., Habib, R., Black, S. E., Szekely, C., Sinden, M., \& Tulving, E. (1997). Cross modality priming in a densely amnesic subject K.C. Brain \& Cognition, 35, 420-423.

Masson, M. E. \& Freedman, L. (1990). Fluent processing of repeated words. Journal of Experimental Psychology: Learning, Memory \& Cognition, 16, 355-373.

McKone, E., \& Slee, J. A. (1997). Explicit contamination in "implicit" memory for new associations. Memory \& Cognition, 25, 352-366.

Moscovitch, M. (1994). Memory and working with memory: Evaluation of a component process model and comparisons with other mod- 
els. In D. Schacter \& E. Tulving (Eds.), Memory systems (pp. 269310). Cambridge, MA: MIT Press.

Perfetti, C. A., \& Bell, L. (1991). Phonological activation during the first $40 \mathrm{msec}$ of word identification: Evidence from backwards masking and priming. Journal of Memory \& Language, 30, 473-485.

Richardson-Klavehn, A., Lee, M. G., Joubran, R, \& BJork, R. A. (1994). Intention and awareness in perceptual identification priming. Memory \& Cognition, 22, 293-312.

Roediger, H. L., III, \& Blaxton, T. A. (1987). Effects of varying modality, surface features, and retention interval on priming in wordfragment completion. Memory \& Cognition, 15, 379-388.

Roediger, H. L., III, \& McDermott, K. B. (1993). Implicit memory in normal human subjects. In F. Boller \& J. Grafman (Eds.), Handbook of neuropsychology (Vol. 8, pp. 63-131). Amsterdam: Elsevier.

RUECKL, J. G., \& MATHEW, S. (1999). Implicit memory for phonological processes in visual stem completion. Memory \& Cognition, 27, 1-11.

Schacter, D. L. (1992). Priming and multiple memory systems: Perceptual mechanisms of implicit memory. Journal of Cognitive Neuroscience, 4, 244-256.
Schacter, D. L., Chiu, C. Y. P., \& Ochsner, K. N. (1993). Implicit memory: A selective review. Annual Review of Neuroscience, 16, 159-182.

Schacter, D. L., \& Church, B. A. (1992). Auditory priming: Implicit and explicit memory for words and voices. Journal of Experimental Psychology: Learning, Memory, \& Cognition, 18, 915-930.

Tulving, E. (1985). How many memory systems are there? American Psychologist, 40, 385-398.

Tulving, E., \& Schacter, D. L. (1990). Priming and human memory systems. Science, 247, 301-305.

Vaidya, C. J., Gabrieli, J. D. E., Keane, M. M., \& Monti, L. A. (1995). Perceptual and conceptual memory processes in global amnesia. Neuropsychology, 9, 580-591.

\section{NOTES}

1. We thank Barbara Church for alerting us to this possibility.

2. This effect was observed in two experiments; however, it only reached the level of significance in one of these experiments.

\section{APPENDIX}

Word and Word Fragment Stimuli

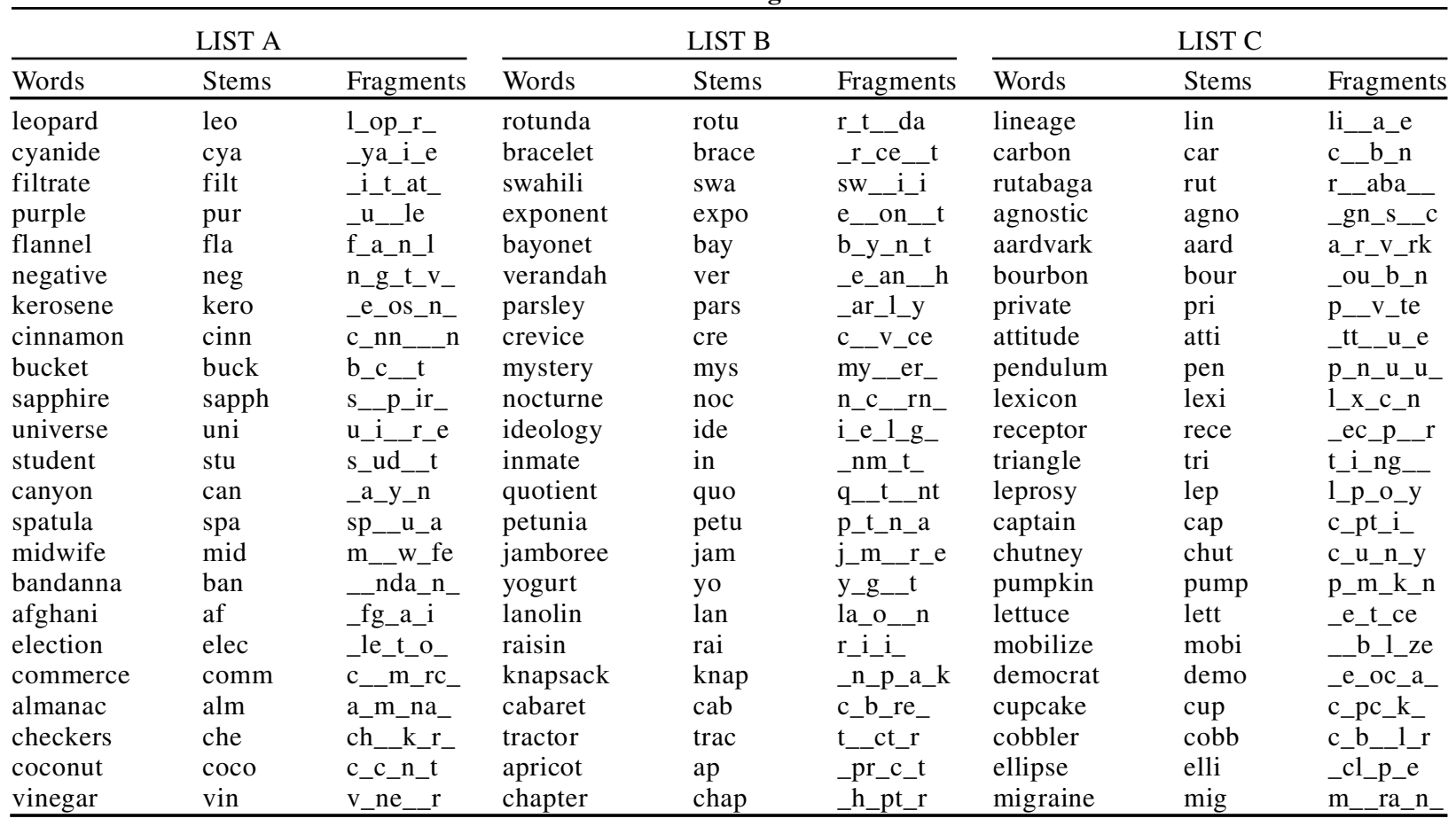

(Manuscript received June 1, 1999;

revision accepted for publication December 10, 1999.) 